\title{
Cell membrane-coated nanoparticles for tumor-targeted drug delivery
}

\author{
Zhilan Chai, Xuefeng $\mathrm{Hu}$ and Weiyue $\mathrm{Lu}^{*}$
}

\begin{abstract}
Nanoparticles can be enriched at tumor site and improve the therapeutic efficacy of many chemotherapy drugs with the well-known enhanced permeability and retention (EPR) effect. While conventional preparations of materials for nanoscale drug delivery system mainly focused on chemical synthesis, recently the combination of synthetic carrier and natural biomimetic carrier has gained more and more attention. As a new generation of biomimetic nanoparticles, cell membrane-coated nanoparticles combine the complex biological functions of natural membranes and the physicochemical properties of synthetic nanomaterials for a more effective drug delivery. Herein, we briefly review the recent advances on cell membrane-coated nanoparticles for tumor-targeted drug delivery via the prolonging systemic circulation lifetime and the active targeting effect. Since the preferential accumulation of cell membrane-coated nanoparticles in tumor site, they are able to improve the therapeutic efficacy of conventional chemotherapy drugs in antitumor treatment as well as to reduce the systemic toxicity. We also introduce a systematic targeted strategy for the promising application of this platform on brain tumors.
\end{abstract}

Keywords: cell membrane, biomimetic nanoparticle, tumor-targeting, drug delivery

\section{INTRODUCTION}

At present, nature-inspired biomimetic delivery systems which combine synthetic materials and natural biomaterials have caught the attention of researchers due to their unique advantages. Coating the intact membranes isolated from cells on the surface of nanoparticles can endow this new nanocarrier with both the physicochemical properties of synthetic nanomaterials and the complicated biological functions of cell membranes. For instance, the red blood cell membrane-coated nanoparticles (RBCNPs) have been developed for detoxification. Loading RBCNPs into hydrogels and the resulting nanosponge could absorb toxins in local bacterial infection treatments [1], while RBCNPs detained staphylococcal $\alpha$-hemolysin ( $\mathrm{Hla}$ ) were also formulated as an anti-virulence vaccine for protection against bacterial pathogens [2]. With the expression of functional membrane-bound acetylcholinesterace on red blood cell membranes (RBC membranes), an anti-organophosphate agent was developed to improve the acetyl cholinesterase (AChE) activity in the blood for systemic organophosphate neutralization [3]. Moreover, utilizing the adhesion antigens and immunomodulatory of the platelets, the platelet membrane coated-nanoparticles enhanced the therapeutic efficacy of docetaxel and vancomycin in coronary restenosis and systemic bacterial infection treatments [4].

Current studies have proved the great potential of the cell membrane-coated nanoparticles in the development of nanoscale drug delivery system in various therapies [5]. In recent years, cancer has been one of the main threats to human health. Presently, most of the anticancer drugs used in clinic do not possess targeting functions [6]. Their simple diffusion and free distribution in human body lead to the lack of tumor specificity and the low drug concentration in tumor site which cause the limited therapeutic efficacy to tumors and serious side effects to normal tissues and organs [7], and therefore reduce the life quality of patients. Still the lack of clinical efficacy and safety remains the main reason of the failure in late-stage clinical trials of most chemotherapy drugs [8].

Compared with conventional pharmaceutical formulations, nanoscale drug delivery system has shown its obvious advantages in improving drug solubility [9], prolonging systemic circulation time [10] and in controlling release of drug [11]. On the one hand, since the existence of EPR effect in tumors [12], nanoparticles are able to enter and concentrate in the tumor site through the pores on

Department of Pharmaceutics, School of Pharmacy, Key Laboratory of Smart Drug Delivery, Fudan University, Shanghai 201203, China

* Corresponding author (email: wylu@shmu.edu.cn) 
tumor angiogenesis and achieve the so-called passive targeting [13]; on the other hand, with the conjugation of targeting molecules like glycosyl $[14,15]$, small molecules [16], antibodies [17], peptides [18-20] on the surface of nanoparticles, they can specifically bind to the target cells and achieve the so-called active targeting. Meanwhile, to decrease the identification of monocytes and macrophages and to prolong the systemic retention time of nanoparticles, hydrophilic polymers such as polyethylene glycol (PEG) are covalently conjugated to the surface of nanoparticles in order to improve the hydrophilicity, flexibility and steric hindrance [21]. However, recent studies have suggested that exogenous substances like PEG possess immunogenicity and that the in vivo clearance rate of PEGylated nanoparticles could be accelerated after multiple doses [22].

Cell membrane-coated nanoparticles can be disguised as endogenous substances, not only reduce reticuloendothelial system uptake [23], but also avoid the immunological recognition [24]. With the targeting capability to source cells of cell membrane itself, active-targeting can be achieved by this platform as well. This review is to illustrate the applications of cell membrane-coated nanoparticles in tumor-targeted drug delivery from its prolonging systemic circulation and tumor-specific targeting effect.

\section{THE PROLONGING SYSTEMIC CIRCULATION OF CELL MEMBRANE- COATED NANOPARTICLES}

The long-circulating nanoparticles can sustainedly release drug in vivo and increase the accumulation of drug in tumor site through the EPR effect [25]. Along with the new developments in molecular and cellular biology, currently scientists have turned their attention from synthetic materials to natural materials.

The red blood cell (RBC) was found to be a natural long-circulating carrier [26], and its unique biological structure and function provide it with the possibility to be designed as a new kind of carrier material [27]. However, due to the complexity of the surface proteins of a biological cell, it is impractical to synthesize all the proteins through conventional chemical methods. Hu et al. [28] have successfully formulated this biomimetic carrier through the separation and extraction of RBC membrane and then coated it onto the surface of nanoparticles. The RBCs are isolated from whole blood and their membranes are obtained after the removal of intracellular components by hypotonic treatment. The membrane vesicles were formed with the collected whole cell membranes through physical extrusion and then are coated onto the poly(lactic-co-gly- colic acid) (PLGA) nanoparticles through mechanical coextrusion and the resulting $\mathrm{RBC}$ membrane-coated nanoparticles (RBCNPs) present a core-shell structure. Surface chemistry study has demonstrated that the RBC membranes are coated onto the surface of nanoparticles in a right-side-out manner to ensure the same performance of molecule interactions of the surface antigens $[29,30]$. Immunohistochemistry studies have shown that the surface antigen density (such as CD47) of RBCNPs was the same as that of RBCs; with respect to PLGA nanoparticles, the uptake by macrophages was reduced by $64 \%$ in vitro, confirming its immune circumvention [31]. Based on a two-compartment model, the RBCNPs possessed a significantly longer systemic circulation than PEGylated PLGA nanoparticles, the elimination half time of RBCNPs was $39.6 \mathrm{~h}$ and that of PEGylated nanoparticles was only 15.8 $\mathrm{h}$ [28]. A similar result was provided by the comparison between gold nanocages (AuNCs) and RBC membrane coated AuNCs (RBC-AuNCs) [32]. The RBC-AuNCs possessed a nearly ten-fold in vivo circulation lifetime than the poly(vinylpyrrolidone)-coated AuNCs. In summary, coating synthetic nanoparticles with cell membranes equip them with the surface proteins of the membranes, leading to the reduction of in vivo clearance rate and a longer circulation time compared to conventional nanoparticles, which is the strength for targeting drug delivery.

Drug loaded RBCNPs have shown an outstanding tumor suppression effect and a lower systemic toxicity as well. Compared to free doxorubicin (Dox), Dox-loaded RBCNPs presented better in vitro stability and sustained drug release, with approximately $85 \%$ of drug released from RBCNPs in comparison to $100 \%$ from PEGylated NPs in $72 \mathrm{~h}$ [33]. A recent study indicated that RBCNPs could deliver Dox to solid tumor site and inhibit the tumor growth in a lymphoma model with excellent in vivo safety and immunocompatibility [34]. The median survival of mice was extended from $24 \mathrm{~d}$ for the isotonic sucrose-treated group to $47 \mathrm{~d}$ for the RBCNPs/Dox-treated group. Hematological parameters showed no abnormality in white blood cell count of RBCNPs/Dox-treated mice while a significant decrease was observed in that of free Dox-treated group. Additionally, no elevation of serum IL-6 level was observed with RBCNPs administration, indicating the lack of acute systemic inflammatory response. Su et al. [35] formulated paclitaxel-loaded RBCNP and co-administrated it with iRGD for antitumor therapy. While taking advantages of the prolonging circulation of RBCNPs and the tumor penetration property of iRGD, the antitumor studies used a metastatic 4T1 breast cancer model and illustrated that the 
tumor growth was inhibited by $90 \%$ and lung metastasis was suppressed by $95 \%$ with lower hematological toxicity. These findings highlight the potential therapeutic efficacy of drug loaded RBCNPs in antitumor treatments since the existence of EPR effect and the sustained drug release in tumor sites.

Overall, the current studies have suggested that RBCNPs possess a prolonged circulation lifetime and sustained drug release property. The resulting enhanced therapeutic efficacy of RBCNPs paired with the reduced systemic toxicity endows this drug delivery system with many essential properties for a clinical transformation.

\section{THE TUMOR-SPECIFIC TARGETING CELL MEMBRANE-COATED NANOPARTICLES}

In order to improve the selectivity and specificity of nanoscale drug delivery system, cell-specific targeting nanocarrier has become the hotspot of tumor therapy. Active targeting drug delivery system has been demonstrated to selectively enter into the tumor cell and results in better therapeutic efficacy [36-38]. For cell membrane-coated nanoparticles, active-targeting can be achieved by introducing targeting ligands through lipid insertion method or by utilizing the targeting capability to source cells of cell membrane itself.

\section{Tumor-specific targeting via lipid insertion}

Currently, chemical synthesis strategies are most widely used to functionalize nanoparticles with targeting ligands, including carboxyl-, amine- and thiol-based chemistry $[39,40]$. However, for cell membrane-coated nanoparticles, other functionalization strategies should be developed instead of chemical synthesis to protect the integrity of carbohydrates and proteins located on the cell membranes. Fang et al. [41] developed a non-destructive strategy to introduce targeting ligands to RBCNPs, which is so-called lipid insertion. Targeting ligands was first conjugated to lipid molecules and then inserted into the RBC membranes. This kind of insertion is allowed by the fluidity and dynamic conformation of the membrane bilayers, therefore it not only protects the surface proteins of RBC membranes but also controls the density of targeting ligands. Researchers successfully decorated small molecules like folic acid $\left(M_{\mathrm{w}}=441 \mathrm{Da}\right)$ and macromolecules like AS1411 $\left(M_{\mathrm{w}}=9000 \mathrm{Da}\right)$ onto the surface of RBCNPs, and the functionalized RBCNPs showed significant targeting effect on KB cells and MCF-7 cells. Fu et al. [42] decorated a tumor-targeting peptide RGD (Arg-Gly-Asp) onto the RBC membrane coated magnetic O-carboxymethyl-chitosan nanoparticle and co-encapsulated two drugs, doxorubincin and paclitaxel, into it. The resulting tumor-targeted nanoparticles were superior to the traditional PEGylated nanoparticles and non-targeted RBC membrane coated nanoparticles in the inhibition of tumor growth since its prolonging circulation and specific cell uptake.

\section{Tumor-specific targeting via cell membrane-coating}

Besides the lipid insertion method, the cell membrane coating itself can also achieve the active targeting because of the intrinsic homotypic or heterotypic adhesion properties of source cells. This kind of natural adhesion plays an important role in biology and can be utilized.

The homologous adhesion domains of the surface antigens on cancer cell membrane like carcinoembtyonic antigen and galectin-3 provided it with the homotypic targeting property [43]. This kind of adhesion property is the key to tumor formation and metastasis [44]. Researchers separated membranes from MDA-MB-435 breast cancer cells and coated them onto the PLGA nanoparticles. The resulted cancer cell membrane-coated nanoparticles were significantly uptaken by MDA-MB-435 cells in vitro, about 40-fold than PLGA NPs and 20-fold than RBCNPs. Moreover, the uptake was specific as there was no significant difference on the uptake by human foreskin fibroblasts cells among these three formulations. This platform colocalized multiple antigens and immunological adjuvants in one particle, which enabled the uptake of membrane-bound tumor antigens for efficient presentation and downstream immune activation [45].

The surface proteins on macrophage cell membrane (MPCM) such as Toll-like receptors and interleukin-1 receptor can specifically recognize the tumor endothelium which enables it to actively target tumor site [46]. Xuan et al. [47] coated MPCMs onto Dox loaded magnetic silica nanoparticles (MSNs) and the resulted MPCM-coated MSNs showed higher toxicity to 4T1 breast cancer cells in vitro, compared to free Dox and Dox loaded silica nanoparticles. The in vivo antitumor study illustrated that the tumor growth of mice with $4 \mathrm{~T} 1$ breast cancer model was effectively inhibited by Dox-loading MPCM-coated MSNs due to the prolonging circulation and active targeting ability. The tumor volume of mice of MPCM-coated MSNs group showed almost no change $15 \mathrm{~d}$ after injection, while those of free Dox group and MSNs group increased by approximately 5.4-fold and 3.5-fold, respectively.

Since the existence of adhesion receptors-mediated 
leukocyte-endothelial cell recognition and interactions [48], the leukocytes possess the capability to bind the inflamed endothelium and the potential to actively recognize tumor endothelium. The leukocyte membrane-coated nanoparticle (LMNP) was developed, and the surface adsorption of protein (IgG and albumin) was decreased and the uptake by macrophages was reduced by $75 \%$. Since LMNP was able to communicate with endothelial cells and transport across the endothelial layer, the accumulation of nanoparticles in tumor site was improved by approximately 2 -fold based on a melanoma model [49].

The biomolecular binding such as P-selectin and CD44 receptors and the structure-based capture [50,51] enable platelets to recognize and interact with circulating tumor cells (CTCs). The aggregation of platelets around CTCs contributes to their survival in blood and leads to tumor metastasis [52-54]. Recently researchers have developed a platelet membrane-coated nanomedicine (PMNP) with TRAIL inserted onto the outer membrane and Dox loaded into the inner nanoparticles. Owing to the interaction between platelet and CTCs, the PMNP specifically delivered TRAIL to cancer cell membrane and activated the extrinsic apoptosis pathway. Meanwhile, Dox accumulation at nuclei was also increased and activated the intrinsic apoptosis pathway. The in vivo antitumor efficacy was studied on MDA-MB-231 breast cancer model and the TRAIL-DoxPMNP group showed the most significant tumor growth inhibition and a slight decrease in lung metastasis, with no obvious toxicity on normal organs observed [55].

Ultimately, lipid insertion method allows RBCNPs to become long-circulating nanoparticles with tumor-targeting effect, while cell membrane (including cancer cell, macrophage, leukocyte and platelet) -coated nanoparticles achieve the same purpose utilizing the biological functions of cell membranes themselves. In either way, this kind of biomimetic nanoparticles have shown great potential for tumor-targeted drug delivery, with improved accumulation in tumor sites and reduced side effects.

\section{CONCLUSIONS AND PROSPECTIVE}

Coating cell membranes onto the surface of nanoparticles in a right-side-out manner endows this new biomimetic nanocarrier with significant potential for tumor-targeting therapy. The membrane maintaining all the lipids, proteins and glycosyls prolongs the circulation time of the nanoparticle and improves the accumulation in tumor site utilizing the biological properties of membrane itself. For precise treatment on tumor, active-targeting strategy has attracted extensive attention in recent decades [56-58].
Through lipid insertion or certain cell membrane coating, the resulted nanoparticles are functionalized with specific targeting property without destruction on membrane proteins and become an active-targeting strategy for a variety of cancers (Fig. 1), including solid tumor cancer (breast cancer and lymphomas) and circulating and metastasis cancer (leukemias). Currently, cell membrane-coating strategy has been rapidly applied on various types of nanostructures, including PLGA nanoparticles [59,60], gold nanoparticles [61,62], silica nanoparticles [47] and gelatin [63]. Towards the future, individual formulations can be customized according to patients' certain treatments in clinic. Since cell membrane-coated nanoparticles maintain the biological function of cell membranes, this platform possesses splendid in vivo safety and biocompatibility which are most regarded in clinical application. Meanwhile, the antitumor effect of this platform has also been proved in various cancer models on mice. Cell membrane-coated nanoparticles, as a new nanotherapeutics with reduced systemic toxicity and enhanced therapeutic efficacy, hold great potential on clinical translation.

Nevertheless, the current researches on cell membrane-coated nanoparticles mainly focus on peripheral system diseases, little advances have been achieved in the central nervous system (CNS) diseases. Primary brain and CNS tumors threaten human health severely since their fast development, poor diagnosis and rapid recurrence. Due to the existence of blood brain barrier (BBB) and the blood brain tumor barrier (BBTB) [64-66], conventional chemotherapy drugs cannot be delivered to brain effectively, which causes few accumulation in tumor sites and severe systematic side effects. In this case, an overall targeting drug delivery system called "systematic targeted drug delivery" has been proposed to be a promising way for the treatments of brain tumors. The "systematic targeted drug delivery" aims at all the potential targets existing in the whole process of brain tumors oncogenesis and development. At the early stage of brain tumors, BBB remains intact and continues to be the key barrier for drug delivery; With the progression of brain tumors, tumor neovasculature has formed and BBTB which locates between tumor tissues and microvessels becomes the main limitation of drug delivery, meanwhile BBB still exists around the tumor edge of infiltrating glioma and impedes the transport of drugs. Additionally, vasculogenic mimicry, the microenvironment of tumor site and tumor stem cells should also be taken into consideration in the design of drug delivery system as potential targets in brain tumor treatment. With successful modification of targeting ligands, "systematic 


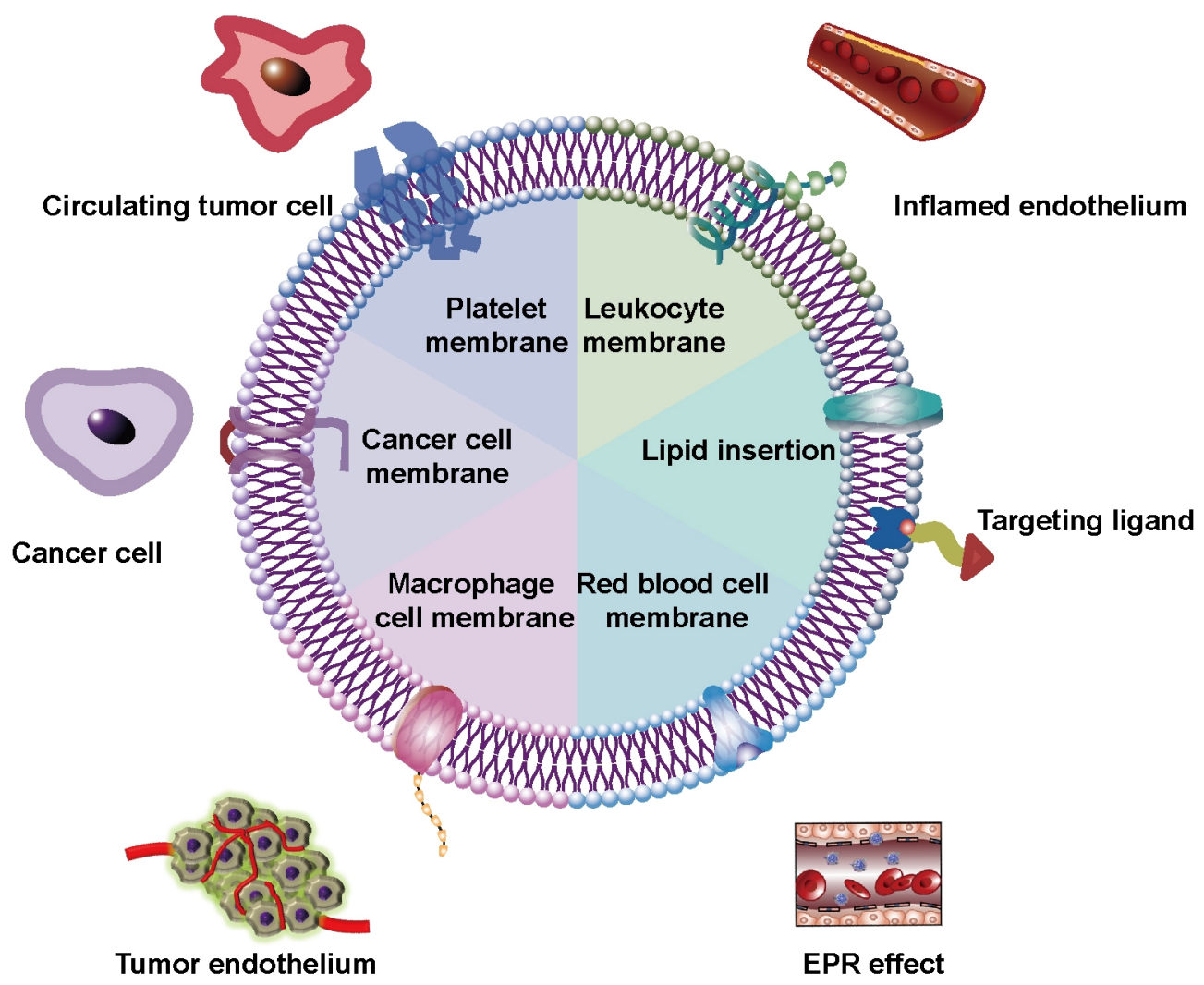

Figure 1 Stategies to "camouflage" nanoparticle for prolonged circulation and active tumor-targeting.

targeted drug delivery" is hoped to be achieved by cell membrane-coated nanoparticles, the obvious less reticuloendothelial system uptake and immunorecognition of this platform also endow it with a longer systematic retention time and more probabilities to traverse multiple barriers in order to release drugs in tumor site accurately and sustainedly. We believe that with the continuous improvements and perfection of the targeting strategies of cell membrane-coated nanoparticles, this platform will hold great potential for a more effective tumor-targeted drug delivery system.

Received 27 November 2016; accepted 20 December 2016; published online 22 January 2017

1 Wang F, Gao W, Thamphiwatana S, et al. Hydrogel retaining toxinabsorbing nanosponges for local treatment of methicillin-resistant Staphylococcus aureus infection. Adv Mater, 2015, 27: 3437-3443

2 Wang F, Fang RH, Luk BT, et al. Nanoparticle-based antivirulence vaccine for the management of methicillin-resistant Staphylococcus aureus skin infection. Adv Funct Mater, 2016, 26: 1628-1635

3 Pang Z, Hu CMJ, Fang RH, et al. Detoxification of organophosphate poisoning using nanoparticle bioscavengers. ACS Nano, 2015, 9: 6450-6458
4 Hu CMJ, Fang RH, Wang KC, et al. Nanoparticle biointerfacing by platelet membrane cloaking. Nature, 2015, 526: 118-121

5 Gao W, Zhang L. Engineering red-blood-cell-membrane-coated nanoparticles for broad biomedical applications. AIChE J, 2015, 61: 738-746

6 Mitragotri S, Burke PA, Langer R. Overcoming the challenges in administering biopharmaceuticals: formulation and delivery strategies. Nat Rev Drug Discov, 2014, 13: 655-672

7 Torchilin VP. Drug targeting. Eur J Pharm Sci, 2000, 11: S81-S91

8 Kola I, Landis J. Opinion: can the pharmaceutical industry reduce attrition rates? Nat Rev Drug Discov, 2004, 3: 711-716

9 Peer D, Karp JM, Hong S, et al. Nanocarriers as an emerging platform for cancer therapy. Nat Nanotech, 2007, 2: 751-760

10 Petros RA, DeSimone JM. Strategies in the design of nanoparticles for therapeutic applications. Nat Rev Drug Discov, 2010, 9: 615-627

11 Torchilin VP. Recent advances with liposomes as pharmaceutical carriers. Nat Rev Drug Discov, 2005, 4: 145-160

12 Matsumura Y, Maeda H. A new concept for macromolecular therapeutics in cancer chemotherapy: mechanism of tumoritropic accumulation of proteins and the antitumor agent smancs. Cancer Res, 1986, 46: 6387-6392

13 Luk BT, Zhang L. Current advances in polymer-based nanotheranostics for cancer treatment and diagnosis. ACS Appl Mater Interfaces, 2014, 6: 21859-21873

14 Cryan SA, Devocelle M, Moran PJ, et al. Increased intracellular targeting to airway cells using octaarginine-coated liposomes: in vitro 
assessment of their suitability for inhalation. Mol Pharm, 2006, 3: 104-112

15 Qin $\mathrm{Y}$, Fan $\mathrm{W}$, Chen $\mathrm{H}$, et al. In vitro and in vivo investigation of glucose-mediated brain-targeting liposomes. J Drug Targeting, 2010, 18: 536-549

16 Dixit V, Van den Bossche J, Sherman DM, et al. Synthesis and grafting of thioctic acid-PEG-folate conjugates onto Au nanoparticles for selective targeting of folate receptor-positive tumor cells. Bioconjugate Chem, 2006, 17: 603-609

17 Manjappa AS, Chaudhari KR, Venkataraju MP, et al. Antibody derivatization and conjugation strategies: application in preparation of stealth immunoliposome to target chemotherapeutics to tumor. J Control Release, 2011, 150: 2-22

18 Wei X, Zhan C, Shen Q, et al. A D-peptide ligand of nicotine acetylcholine receptors for brain-targeted drug delivery. Angew Chem, 2015, 127: 3066-3070

19 Yan Z, Wang F, Wen Z, et al. LyP-1-conjugated PEGylated liposomes: a carrier system for targeted therapy of lymphatic metastatic tumor. J Controlled Release, 2012, 157: 118-125

20 Sun X, Pang Z, Ye H, et al. Co-delivery of pEGFP-hTRAIL and paclitaxel to brain glioma mediated by an angiopep-conjugated liposome. Biomaterials, 2012, 33: 916-924

21 Harris JM, Chess RB. Effect of pegylation on pharmaceuticals. Nat Rev Drug Discov, 2003, 2: 214-221

22 Knop K, Hoogenboom R, Fischer D, et al. Poly(ethylene glycol) in drug delivery: pros and cons as well as potential alternatives. Angew Chim Int Ed, 2010, 49: 6288-6308

23 Rao L, Xu JH, Cai B, et al. Synthetic nanoparticles camouflaged with biomimetic erythrocyte membranes for reduced reticuloendothelial system uptake. Nanotechnology, 2016, 27: 085106

24 Théry C, Ostrowski M, Segura E. Membrane vesicles as conveyors of immune responses. Nat Rev Immunol, 2009, 9: 581-593

25 Davis ME, Chen ZG, Shin DM. Nanoparticle therapeutics: an emerging treatment modality for cancer. Nat Rev Drug Discov, 2008, 7: 771-782

26 Merkel TJ, Jones SW, Herlihy KP, et al. Using mechanobiological mimicry of red blood cells to extend circulation times of hydrogel microparticles. Proc Natl Acad Sci USA, 2011, 108: 586-591

27 Doshi N, Zahr AS, Bhaskar S, et al. Red blood cell-mimicking synthetic biomaterial particles. Proc Natl Acad Sci USA, 2009, 106: 21495-21499

28 Hu CMJ, Zhang L, Aryal S, et al. Erythrocyte membrane-camouflaged polymeric nanoparticles as a biomimetic delivery platform. Proc Natl Acad Sci USA, 2011, 108: 10980-10985

29 Luk BT, Jack Hu CM, Fang RH, et al. Interfacial interactions between natural RBC membranes and synthetic polymeric nanoparticles. Nanoscale, 2014, 6: 2730-2737

30 Fan Z, Zhou H, Li PY, et al. Structural elucidation of cell membrane-derived nanoparticles using molecular probes. J Mater Chem B, 2014, 2: 8231-8238

31 Hu CMJ, Fang RH, Luk BT, et al. "Marker-of-self” functionalization of nanoscale particles through a top-down cellular membrane coating approach. Nanoscale, 2013, 5: 2664-2668

32 Piao JG, Wang L, Gao F, et al. Erythrocyte membrane is an alternative coating to polyethylene glycol for prolonging the circulation lifetime of gold nanocages for photothermal therapy. ACS Nano, 2014, 8: 10414-10425

33 Aryal S, Hu CMJ, Fang RH, et al. Erythrocyte membrane-cloaked polymeric nanoparticles for controlled drug loading and release. Nanomedicine, 2013, 8: 1271-1280

34 Luk BT, Fang RH, Hu CMJ, et al. Safe and immunocompatible nanocarriers cloaked in $\mathrm{RBC}$ membranes for drug delivery to treat solid tumors. Theranostics, 2016, 6: 1004-1011

35 Su J, Sun H, Meng Q, et al. Long circulation red-blood-cellmimetic nanoparticles with peptide-enhanced tumor penetration for simultaneously inhibiting growth and lung metastasis of breast cancer. Adv Funct Mater, 2016, 26: 1243-1252

36 Xiong XB, Huang Y, Lu WL, et al. Intracellular delivery of doxorubicin with RGD-modified sterically stabilized liposomes for an improved antitumor efficacy: in vitro and in vivo. J Pharm Sci, 2005, 94: $1782-1793$

37 Farokhzad OC. Using ligands to target cancer cells. Clin Adv Hematol Oncol, 2012, 10: 543-544

38 Ying M, Shen Q, Liu Y, et al. Stabilized heptapeptide A7R for enhanced multifunctional liposome-based tumor-targeted drug delivery. ACS Appl Mater Interfaces, 2016, 8: 13232-13241

39 Gu F, Zhang L, Teply BA, et al. Precise engineering of targeted nanoparticles by using self-assembled biointegrated block copolymers. Proc Natl Acad Sci USA, 2008, 105: 2586-2591

40 Wei X, Gao J, Zhan C, et al. Liposome-based glioma targeted drug delivery enabled by stable peptide ligands. J Controlled Release, 2015, 218: 13-21

41 Fang $\mathrm{RH}, \mathrm{Hu} \mathrm{CMJ}$, Chen $\mathrm{KNH}$, et al. Lipid-insertion enables targeting functionalization of erythrocyte membrane-cloaked nanoparticles. Nanoscale, 2013, 5: 8884-8888

42 Fu Q, Lv P, Chen Z, et al. Programmed co-delivery of paclitaxel and doxorubicin boosted by camouflaging with erythrocyte membrane. Nanoscale, 2015, 7: 4020-4030

43 Khaldoyanidi SK, Glinsky VV, Sikora L, et al. MDA-MB-435 human breast carcinoma cell homo- and heterotypic adhesion under flow conditions is mediated in part by Thomsen-Friedenreich antigen-galectin-3 interactions. J Biol Chem, 2003, 278: 4127-4134

44 Glinsky VV, Glinsky GV, Glinskii OV,et al. Intravascular metastatic cancer cell homotypic aggregation at the sites of primary attachment to the endothelium. Cancer Res, 2003, 63: 3805-3811

45 Fang RH, Hu CMJ, Luk BT, et al. Cancer cell membrane-coated nanoparticles for anticancer vaccination and drug delivery. Nano Lett, 2014, 14: 2181-2188

46 lpage $\{415\}$, . Kawahara . Infiltration of thymidine phosphorylasepositive macrophages is closely associated with tumor angiogenesis and survival in intestinal type gastric cancer. Oncol Rep, 2010, 24: 405

47 Xuan M, Shao J, Dai L, et al. Macrophage cell membrane camouflaged mesoporous silica nanocapsules for in vivo cancer therapy. Adv Healthcare Mater, 2015, 4: 1645-1652

48 Butcher EC. Leukocyte-endothelial cell recognition: three (or more) steps to specificity and diversity. Cell, 1991, 67: 1033-1036

49 Parodi A, Quattrocchi N, van de Ven AL, et al. Synthetic nanoparticles functionalized with biomimetic leukocyte membranes possess cell-like functions. Nat Nanotech, 2012, 8: 61-68

50 Borsig L, Wong R, Feramisco J, et al. Heparin and cancer revisited mechanistic connections involving platelets, P-selectin, carcinoma mucins, and tumor metastasis. Proc Natl Acad Sci USA, 2001, 98: 3352-3357

51 Labelle M, Begum S, Hynes RO. Platelets guide the formation of early metastatic niches. Proc Natl Acad Sci USA, 2014, 111: E3053-E3061

52 Nash G, Turner L, Scully M, et al. Platelets and cancer. Lancet Oncol, 2002, 3: 425-430

53 Chaffer CL, Weinberg RA. A perspective on cancer cell metastasis Science, 2011, 331: 1559-1564

54 Gay LJ, Felding-Habermann B. Platelets alter tumor cell attributes to propel metastasis: programming in transit. Cancer Cell, 2011, 20: $553-554$ 
55 Hu Q, Sun W, Qian C, et al. Anticancer platelet-mimicking nanovehicles. Adv Mater, 2015, 27: 7043-7050

56 Li Y, Lin J, Huang Y, et al. Self-targeted, shape-assisted, and controlled-release self-delivery nanodrug for synergistic targeting/anticancer effect of cytoplasm and nucleus of cancer cells. ACS Appl Mater Interfaces, 2015, 7: 25553-25559

57 Li Y, Lin J, Wu H, et al. Orthogonally functionalized nanoscale micelles for active targeted codelivery of methotrexate and mitomycin C with synergistic anticancer effect. Mol Pharm, 2015, 12: 769-782

58 Ying M, Zhan C, Wang S, et al. Liposome-based systemic gliomatargeted drug delivery enabled by all-D peptides. ACS Appl Mater Interfaces, 2016, 8: 29977-29985

59 Hu CMJ, Fang RH, Copp J, et al. A biomimetic nanosponge that absorbs pore-forming toxins. Nat Nanotech, 2013, 8: 336-340

60 Copp JA, Fang RH, Luk BT, et al. Clearance of pathological antibodies using biomimetic nanoparticles. Proc Natl Acad Sci USA, 2014, 111: 13481-13486

61 Gao W, Fang RH, Thamphiwatana S, et al. Modulating antibacterial immunity via bacterial membrane-coated nanoparticles. Nano Lett, 2015, 15: 1403-1409

62 Xuan M, Shao J, Dai L, et al. Macrophage cell membrane camouflaged au nanoshells for in vivo prolonged circulation life and en- hanced cancer photothermal therapy. ACS Appl Mater Interfaces, 2016, 8: 9610-9618

63 Zhang J, Gao W, Fang RH, et al. Synthesis of nanogels via cell membrane-templated polymerization. Small, 2015, 11: 4309-4313

64 Daneman R. The blood-brain barrier in health and disease. Ann Neurol, 2012, 72: 648-672

65 Wong AD, Ye M, Levy AF, et al. The blood-brain barrier: an engineering perspective. Front Neuroeng, 2013, 6: 7

66 Ningaraj NS. Regulation of blood-brain tumor barrier permeability by calcium-activated potassium channels. J Pharm Exp Ther, 2002, 301: $838-851$

Acknowledgments This work was supported by the National Basic Research Program of China (2013CB932500), and the National Natural Science Foundation of China (81273458 and 81473149).

Author contributions $\mathrm{Lu} \mathrm{W}$ proposed the review of tumor-targeted cell membrane-coated nanoparticles. Chai $\mathrm{Z}$ investigated literatures and wrote the paper. All authors contributed to the general discussion.

Conflict of interest The authors declare that they have no conflict of interest.

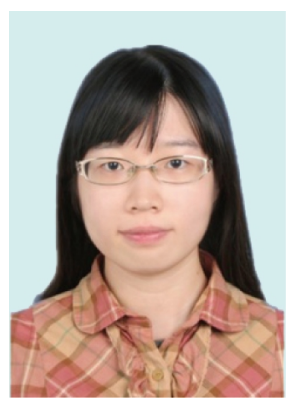

Zhilan Chai is a graduate student from the Department of Pharmaceutics, School of Pharmacy, Fudan University. She got her BCs degree in pharmacy from Fudan University (2011). In 2015, she joined Prof. Weiyue Lu's group for further study. Her current research interests focus on red blood cell membrane coated-nanoparticle and its brain-targeting strategy.

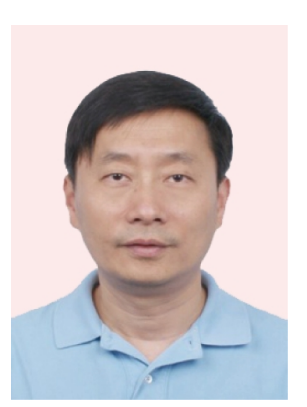

Weiyue Lu is a professor at the Department of Pharmaceutics, School of Pharmacy, Fudan University, the Director of Key laboratory of Smart Drug Delivery (Fudan University), Ministry of Education, China, the Principle Investigator of National Basic Research Program (973), and the Director of the Pharmaceutics Committee and Associate Director of the Nanomedicine Committee of Chinese Pharmaceutical Association (CPA). Professor Lu's research focuses on brain, lymphatic system and its tumor targeting strategy and drug delivery system.

\title{
用于肿瘤靶向递药的细胞膜包覆纳米粒子
}

\author{
柴芝兰, 胡雪峰, 陆伟跃* \\ 摘要 由于EPR效应, 纳米粒子能够在肿瘤部位浓集并提高许多化疗药物的治疗指数. 传统纳米递药系统的载体材料主要通过化学合成方 \\ 法制备, 而目前将化学合成载体与天然仿生载体相结合的策略得到越来越多的关注. 细胞膜包覆纳米粒子作为新一代仿生纳米制剂, 它将 \\ 细胞膜特有的生物学功能与化学合成材料的理化性质相结合, 形成更有效的递药系统. 本文就细胞膜包覆纳米粒子的长循环效果及主动 \\ 靶向作用在肿瘤靶向治疗中的研究进行综述, 并就其在脑部肿瘤治疗中的应用前景进行了展望.
}

